\title{
Multidetector cardiac tomography: A useful tool before cardiac resynchronization therapy
}

\author{
Maria de la Paz Ricapito ${ }^{1}$, Diego Conde ${ }^{2}$, Marie M. Theriault ${ }^{1}$, \\ Santiago Rivera ${ }^{1}$, Mariano G. Badra-Verdu ${ }^{1}$, Jean F. Roux ${ }^{1}$, Paul Farand ${ }^{1}$, \\ Félix A. Ayala-Paredes ${ }^{1}$, Gerald Gahide ${ }^{1}$ \\ ${ }^{1}$ Centre Hospitalier Universitaire de Sherbrooke, Quebec, Canada \\ ${ }^{2}$ Instituto Cardiovascular de Buenos Aires, Buenos Aires, Argentina
}

\begin{abstract}
Background: Left ventricular lead placement in a suitable coronary vein is a key determinant of responsiveness to cardiac resynchronization therapy (CRT). Multidetector cardiac tomography (MDCT) is a non-invasive alternative to depict cardiac venous anatomy although coronary sinus $(C S)$ retrograde venography $(R V)$ is the gold standard. The aim of this study was to evaluate the accuracy of MDCT to determine the presence of CS tributaries before CRT. Methods: A retrospective analysis of 41 consecutive patients eligible to CRT was performed. $M D C T$ was assessed in all patients before CRT and RV was achieved in 39 patients. Both methods evaluated the presence of the inferior interventricular vein (IIV), posterior vein (PV) and lateral main vein (LMV). CS ostium diameter and distance between the CS ostium and right atrium (RA) lateral wall were also measured.

Results: The IIV was identified in $100 \%$ of MDCT and in $43.6 \%$ of RV. In comparison to RV, the MDCT's sensitivity to identify PV and LMV was $100 \%$ for both, kappa coefficient of 0.792 (CI 95\% 0.46-0.93) and 0.69 (CI 95\% 0.46-0.91), respectively. There was no significant difference between ischemic and non-ischemic patients regarding the presence of PV or LMV. Median CS antero-posterior diameter was $10.3 \mathrm{~mm}$ (IQR 7.5-13) and supero-inferior was $14.1 \mathrm{~mm}$ $(I Q R$ 11.5-17) $(p<0.01)$. A positive correlation $(p<0.001)$ between echocardiographic $R A$ area and the distance from CS ostium to the RA lateral wall in the MDCT was observed.
\end{abstract}

Conclusions: MDCT is as accurate as RV to depict CS and its tributaries (IIV, PV, LMV), and it could be useful as a non-invasive technique before CRT. (Cardiol J 2015; 22, 5: 590-596)

Key words: multidetector computed tomography, resynchronization therapy, cardiomyopathy, cardiac veins, pacemaker, heart failure

\section{Introduction}

Current cardiac guidelines support cardiac resynchronization therapy (CRT) for symptomatic heart failure (HF) patients (New York Heart Association functional [NYHA] class III or IV) on optimal pharmacologic therapy, QRS $\geq 120 \mathrm{~ms}$ and left ven- tricular ejection fraction $(\mathrm{LVEF}) \leq 35 \%$ [1]. CRT may be considered in patients with class II HF, and even class I with QRS > $150 \mathrm{~ms}$ according to the $2013 \mathrm{ACCF} / \mathrm{AHA}$ guidelines for the management of HF [2]. CRT improves symptoms, functional status, ventricular size and function, hospitalization rate and mortality in patients with advanced HF [3].

Address for correspondence: Diego Conde, MD, Blanco Encalada 1543, Buenos Aires, Zip Code 1428, Argentina, tel: +54 9116381 6339, fax: +54 114787 7500, ext.3170, e-mail: drconde@hotmail.com 
However, approximately $25-30 \%$ of patients who meet standard criteria for CRT fails to derive substantial benefits [4]. Left ventricular (LV) lead placement in a suitable cardiac vein branch along the dyssynchronous wall of the $\mathrm{LV}$ is an important determinant of responsiveness to CRT [5]. As most of those patients have cardiomegaly, the coronary sinus (CS) is larger and its anatomic location may be displaced. Implanting the electrode in a cardiac vein can be difficult in as many as $20 \%$ of patients, a consequence of unfavorable anatomic factors. In such patients, accurate anatomic depiction of the vessels can be crucial to the success of LV pacing [6]. Retrograde venography (RV) via CS is currently the standard technique for defining CS anatomy [5]. Ideally, venous anatomy should be assessed noninvasively before implantation to determine whether a transvenous approach is feasible [7]. Cardiac computed tomography (CT) has been increasingly used to assess the anatomy of cardiac vessels, including the cardiac venous system (CVS) [7]. The feasibility of multidetector cardiac tomography (MDCT) to visualize the venous anatomy has been demonstrated [7-11]. It has the advantage of showing the entire CS and the origin of the inferior interventricular vein (IIV), which is obscured by the balloon in RV. With MDCT, there is no foreshortening or vessel overlap. It has also been demonstrated that MDCT properly depicts variants of normal anatomy and small branches [6].

The objective of the present study was to evaluate the accuracy of 320 detectors CT scan to depict cardiac veins and right atrium (RA) anatomy before CRT.

\section{Methods}

\section{Study design}

This was a single center retrospective study. The study conforms to the principles of Helsinki declaration and was approved by the hospital's Ethics Committee. Given the retrospective character of this study, the ethics committee waived the necessity for informed consent. All authors have read and agreed to the manuscript as written.

\section{Study population}

A cohort of consecutive patients referred to CRT from January to November 2012 was studied. All patients were under optimal medical treatment, with NYHA class III or IV symptoms, LVEF $\leq 35 \%$ and $\mathrm{QRS} \geq 120 \mathrm{~ms}$. A creatinine clearance $\leq 30 \mathrm{~mL} / \mathrm{min}$, allergy to iodinated contrasts, resting heart rate $>100 \mathrm{bpm}$ were considered as contraindications for performing the MDCT study and those patients were excluded from the study. The population was divided into two groups regarding ischemic (IS) or non-ischemic (NIS) cardiomyopathy to allow a sub-analysis.

\section{MDCT}

Scan protocol. Images were taken with a Toshiba Aquilion 320 detector Tomographer (Toshiba Medical Systems, Otawara, Japan) less than 30 days before the CRT. Scanning coverage was from the level of the carina to the bottom of the heart. One hundred milliliters of iodine contrast $\left(\right.$ Visipaque $^{\mathrm{R}}$ ) were administered at $5 \mathrm{~mL} / \mathrm{s}$, followed by a $50 \mathrm{~mL}$ saline flush. The acquisition was performed when the peak density reached 200 $\mathrm{HU}$ in the descending aorta and was prospectively synchronized with the electrocardiogram (ECG) to lower radiation exposure. Scanning was done with collimation: $0.5 \mathrm{~mm}$, tube rotation time: $400 \mathrm{~ms}$, tube voltage: from 100 to $135 \mathrm{kV}$ and 300 to $510 \mathrm{mAs}$, according to the patient's body mass index.

Image reconstruction. ECG-gated acquisitions were reformatted at $75 \%$ of the R-R interval. Aquarius Intuition 4.4 (TeraRecon Inc. Berlin, Germany) was used to make data post-processing.

Qualitative and quantitative evaluation of MDCT data. Images were evaluated by 2 physicians experienced in cardiac imaging (MPR 5y, GG 12y). The following CS tributaries: IIV, posterior vein (PV) of the LV and left marginal vein (LMV) were identified on volume rendered reformations. Multiplanar reformatting in 3 orthogonal planes was used to measure antero-posterior and superoinferior CS ostium diameters (where CS makes an angle with the RA in the crux cordis area). The RA area and the distance between CS ostium and RA lateral wall were also measured.

\section{Retrograde venography}

Venography protocol. Following the CS cannulation, a retrograde angiography was performed using a balloon occlusion catheter and hand injection of contrast. If any veins demonstrated on the MDCT were not encountered on venography, the procedure was repeated moving the catheter upstream and down-stream to find all veins. Fluoroscopic acquisition was continued several seconds after the end of contrast injection to allow retrograde filling of branches occluded by the balloon via collateral flow. Two orthogonal views (right anterior oblique [RAO] $30-45^{\circ}$ and left anterior oblique [LAO] $30-45^{\circ}$ ) were performed for a better 
visualization of the venous tree and appropriate targeting of the LV lead.

Qualitative data in retrograde venography. Venographies were retrospectively reviewed by 2 electrophysiologists to determine the presence of CS tributaries: IIV, PV and LMV.

\section{Statistical analysis}

Each categorical variable is expressed as a percentage. The corresponding number of patients is indicated. Variables are presented as medians with respective inter-quartile ranges (IQR). SPSS 16.0 (Chicago, Illinois, USA) was used for statistical analysis. The sensitivity of MDCT for depicting CS tributaries was determined in comparison to RV. A p value $<0.05$ was considered statistically significant. Inter-observer agreement was calculated using the intra-class coefficient.

\section{Results}

\section{Patients' demographics}

A total of 41 patients were included. MDCT was successfully performed in those patients. RV was not obtained in 2 patients, because of a giant $\mathrm{RA}$, too dilated to properly cannulate the CS in 1 case and because of a right subclavian vein occlusion in the other case.

The median age of the population was 70 years (IQR 66-74 years) and $75.61 \%(\mathrm{n}=31)$ were males. All patients $(100 \%)$ were with NYHA class III and $97.6 \%(\mathrm{n}=40)$ were under beta-blockers. The median LVEF was 24\% (IQR 20-25\%). Population baseline characteristics are summarized in Tables 1 and 2 .

\section{MDCT analysis}

The median antero-posterior and superoinferior diameters CS's ostium were $10.3 \mathrm{~mm}$ (IQR 7.5-13 mm) and $14.1 \mathrm{~mm}$ (IQR 11.5-17 mm), respectively. There was a statistically significant difference between antero-posterior and superoinferior diameters $(\mathrm{p}<0.001)$ indicating an asymmetrical, ovoid shape of the CS ostium (Fig. 1). The median RA area was $21.6 \mathrm{~cm}^{2}$ (IQR $17.5-26.9 \mathrm{~cm}^{2}$ ).

There was a good inter-observer agreement for the measures of CS's ostium antero-posterior diameters $(r=0.83 ; \mathrm{p}<0.001)$, supero-inferior diameters $(\mathrm{r}=0.84 ; \mathrm{p}<0.001)$ and $\mathrm{RA}$ areas $(r=0.92 ; p<0.001)$.

The median of the distance between CS ostium and RA lateral wall was $49 \mathrm{~mm}$, (IQR $44.3-58.2 \mathrm{~mm}$ ) and other atrial measures are summarized at Table 3 . A statistically significant positive correla-
Table 1. Population baseline characteristics.

\begin{tabular}{lc}
\hline Population characteristics & Patients \\
\hline Males & $75.6 \%$ \\
High blood pressure & $78.0 \%$ \\
Dyslipidemia & $80.4 \%$ \\
Type II diabetes 31.7\% & \\
Previous pacemaker & $53.7 \%$ \\
Previous implantable cardioverter- & $31.7 \%$ \\
-defibrillator & \\
New York Heart Association class III & $100 \%$ \\
Beta-blockers & $97.6 \%$ \\
Ischemic cardiomyopathy & $53.4 \%$ \\
Non-ischemic cardiomyopathy & $47.6 \%$ \\
\hline
\end{tabular}

Table 2. Echocardiographic measures.

\begin{tabular}{lc}
\hline Echocardiographic parameters & Median (range) \\
\hline Ejection fraction & $24(15-34)$ \\
Right atrium area $\left[\mathrm{cm}^{2}\right]$ & $17(15-21)$ \\
Left atrium area $\left[\mathrm{cm}^{2}\right]$ & $20(17-33)$ \\
LVED diameter $[\mathrm{mm}]$ & $62(57-65)$ \\
LVES diameter $[\mathrm{mm}]$ & $54(45-56)$ \\
LVM index $\left[\mathrm{g} / \mathrm{m}^{2}\right]$ & $112(111-154)$ \\
\hline
\end{tabular}

LVED — left ventricular end diastolic; LVES — left ventricular end systolic; LVM - left ventricular mass

tion ( $\mathrm{r}=0.749 ; \mathrm{p}<0.001)$ between echocardiographic RA area and the distance from CS ostium to the RA lateral wall in the MDCT, was observed.

Regarding the presence of CS tributaries, the IIV was demonstrated in all patients, draining into the CS. In $4.9 \%(n=2)$ of patients, the IIV was duplicated and in $2.4 \%(\mathrm{n}=1)$ the IIV had a common ostium with the PV. The PV was demonstrated in $73.2 \%(\mathrm{n}=30)$ of patients and $12.2 \%(\mathrm{n}=5)$ of patients had a second PV. The LMV was observed in $56.1 \%(\mathrm{n}=23)$.

\section{Venography analysis}

RV was not successfully performed in 2 cases as previously indicated. The IIV was observed in 43.6\% ( $\mathrm{n}=17$ ) while $\mathrm{PV}$, second PV and LMV were depicted in $64.1 \%(\mathrm{n}=25), 10.3 \%(\mathrm{n}=4)$ and $53.8 \%(\mathrm{n}=21)$, respectively.

\section{Comparison between MDCT and RV}

The capability of MDCT for depicting the presence of CS tributaries was systematically compared to RV in 39 patients (Fig. 2). The IIV 


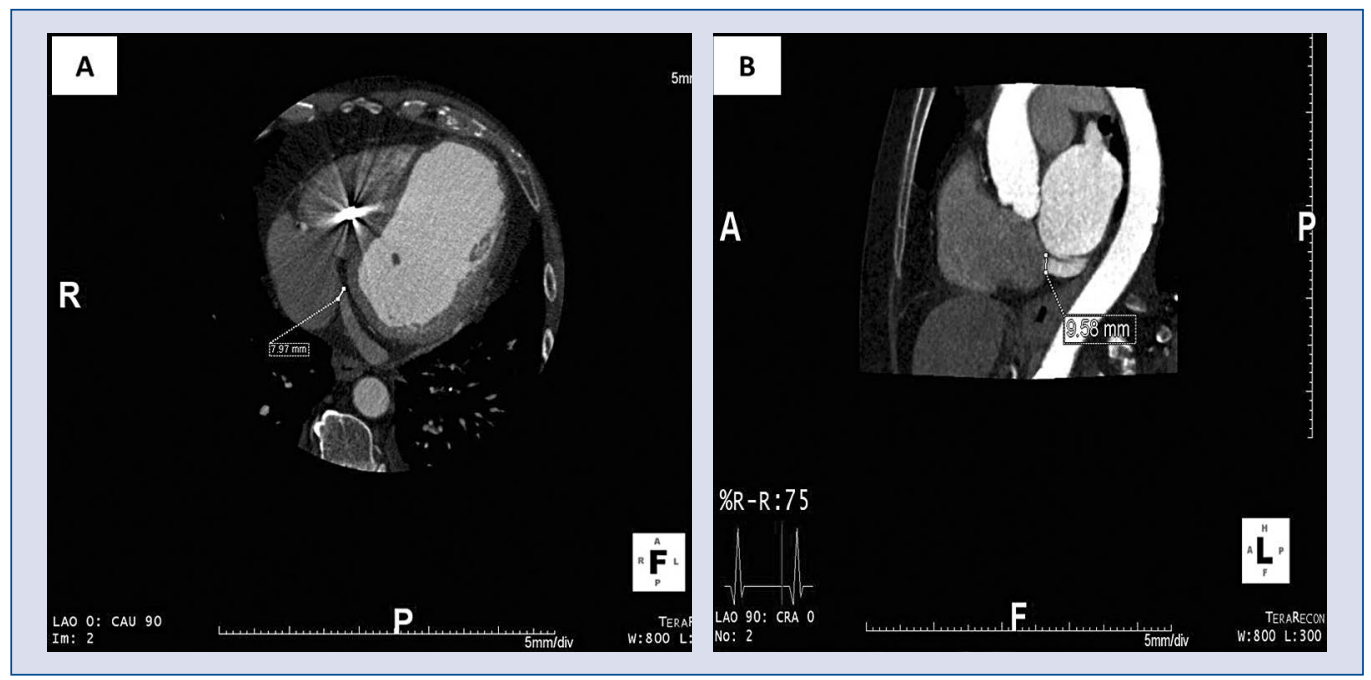

Figure 1. Multidetector cardiac tomography images of the coronary sinus showing its ostium asymmetry; A. Coronary sinus antero-posterior diameter; B. Coronary sinus supero-inferior diameter.

Table 3. Multidetector cardiac tomography (MDCT) measures of coronary sinus (CS) and right atrium (RA).

\begin{tabular}{lcc}
\hline MDCT parameters & Median & $\begin{array}{c}\text { Interquartile } \\
\text { range }\end{array}$ \\
\hline $\begin{array}{l}\text { Antero-posterior } \\
\text { CS diameter [mm] }\end{array}$ & 10.3 & $7.5-13$ \\
$\begin{array}{l}\text { Supero-inferior } \\
\text { CS diameter [mm] }\end{array}$ & 14.1 & $11.5-17$ \\
$\begin{array}{l}\text { RA area [mm }{ }^{2} \text { ] } \\
\begin{array}{l}\text { Distance from CS } \\
\text { to RA lateral wall [mm] }\end{array}\end{array}$ & 21.6 & $17.5-26.9$ \\
\hline
\end{tabular}

was observed on all MDCT but only in $43.6 \%$ $(\mathrm{n}=17)$ of RV. PV was present in $64.1 \%(\mathrm{n}=25)$ of patients according to both exams. In 5 additional patients, PV was observed only on MDCT. The sensitivity of MDCT for detecting PV was $100 \%$, with a kappa coefficient of 0.7 (95\% CI 0.46-0.93). A second PV was depicted in $12.8 \%(n=5)$ of patients on MDCT and in $10.25 \%(\mathrm{n}=4)$ on RV. The sensitivity of MDCT was $100 \%$, with a kappa coefficient of 0.87 (95\% CI 0.63-1.12). The LMV was depicted on MDCT and RV in 19 patients. MDCT's sensitivity was $100 \%$ with a kappa coefficient 0.69 (95\% CI 0.46-0.91).

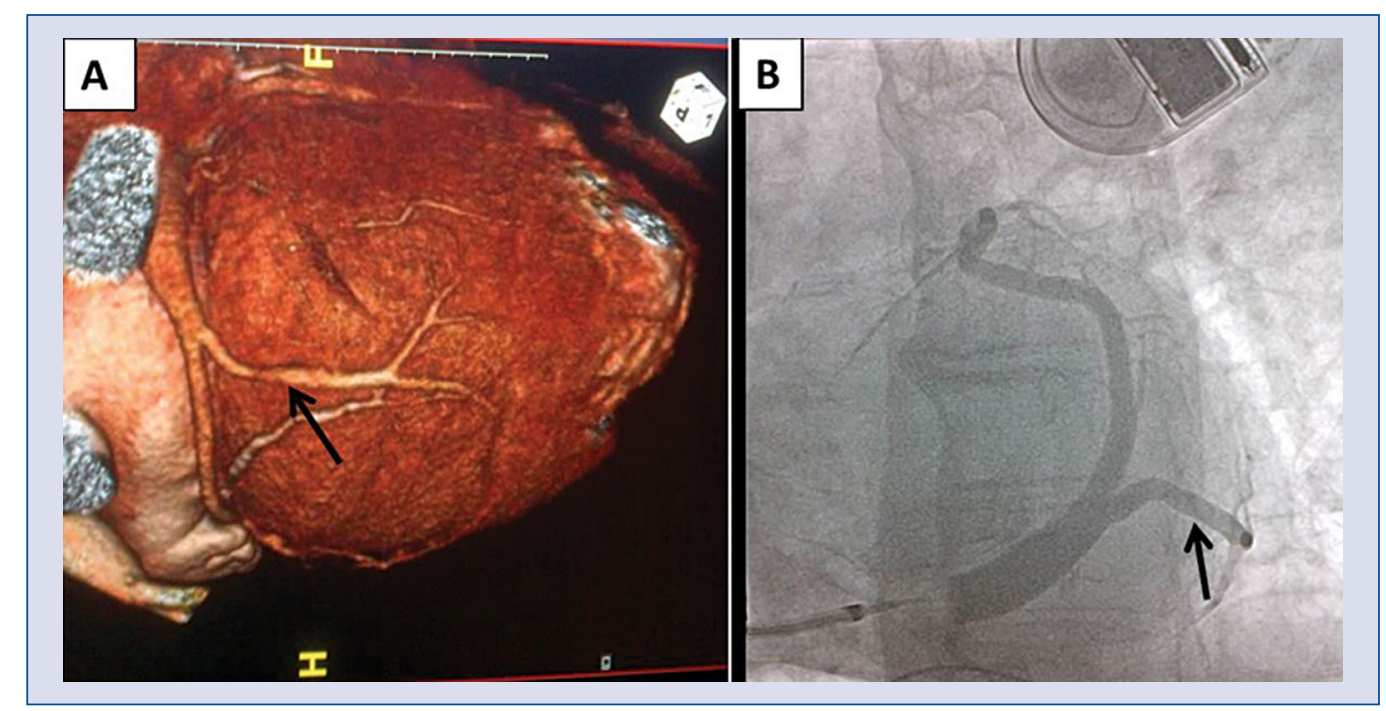

Figure 2. Image of lateral vein in multidetector cardiac tomography 3-dimensional volume renders reconstruction (A) and the same artery in right ventricular projection (B). 


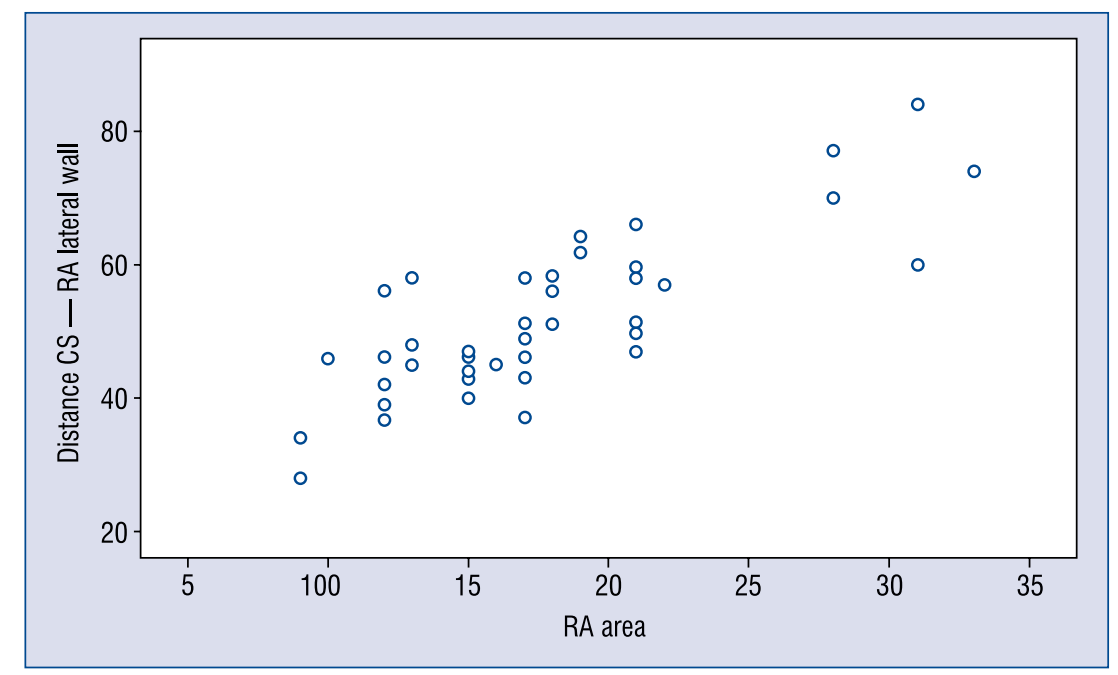

Figure 3. This graphic shows the positive correlation between the distance from coronary sinus (CS) ostium to lateral right atrium (RA) wall in multidetector cardiac tomography and left atrium area measured by echocardiography.

\section{Ischemic versus non-ischemic patients}

Among the 39 patients examined with both techniques, $53.8 \%(\mathrm{n}=21)$ were IS and $46.2 \%(\mathrm{n}=18)$ were NIS. The PV was demonstrated in $66.7 \%$ $(n=12)$ of NIS patients and $61.9 \%(n=13)$ of IS patients on MDCT. The LMV was demonstrated on MDCT in $66.7 \%(\mathrm{n}=12)$ of NIS and $42.9 \%(\mathrm{n}=9)$ of IS. There was no significant difference between IS and NIS patients regarding the presence of PV $(\mathrm{p}=0.76)$ or $\operatorname{LMV}(\mathrm{p}=0.14)$.

\section{Comparison of MDCT and echocardiography}

There was a positive correlation between the RA area and the distance from the lateral wall to the CS ostium measured on MDCT $(r=0.76$; $\mathrm{p}<0.001$ ) (Fig. 3). We also demonstrated a positive correlation between RA area measured in echocardiography and $\operatorname{MDCT}(r=0.83 ; p<0.001)$.

\section{Discussion}

CRT is one of the most successful HF therapies that emerged in the last 25 years and is applicable to almost 25-30\% of patients with symptomatic HF. Since the initial approval of the therapy over 10 years ago, there have been hundreds of thousands of procedures worldwide. Historically, significant attention has been paid to the technical aspects of the implant procedure, particularly regarding the placement of the LV lead. Placement of the transvenous epicardial LV lead is critical to achieving cardiac resynchronization and to garnering the dramatic improvement in symptoms, quality of life, LV func- tion, hospitalization and mortality rates in patients with systolic dysfunction, QRS delay, and HF [12].

The optimal site for LV lead implantation may vary, depending on the region and/or extent of dyssynchrony. CVS anatomy is very variable and there may not always be a suitable vein that can accommodate a pacing lead with acceptable pacing parameters [13].

Identifying patients who do not have a suitable vein for CRT lead implantation is mandatory. These patients may best be treated with an epicardial lead implantation that requires a minimal surgical approach [10, 14, 15].

Cardiac veins have a macroscopic disposition different from that of the coronary arteries and show many more variations [6].

Recent anatomic classification divides the cardiac veins into two main groups: tributaries of the greater CVS and tributaries of the lesser CVS. The greater CVS is subdivided into two groups: CS and non-CS tributaries. The CS tributaries include the CS, great cardiac vein, LMV, PV, and IIV [6]. The left ventricle and atrium and part of the right ventricle are drained by CS tributaries. Most of the right ventricle and both atria are drained by non-CS tributaries.

The CS has become a clinically important structure especially by providing access for different cardiac procedures. Cannulation of the CS may be complicated by obstruction due to Thebesian valve $[16,17]$.

Until recently, exploring the CVS necessitated an invasive approach using RV, either by manual 
contrast injection or after occlusion of the CS $[7,10,18,19]$.

In most patients, CVS anatomy using RV is properly depicted and venous accessibility determined. Reported disadvantages of this method are incomplete venous system visualization, a difficult CS ostium cannulation, an excessive use of contrast material (up to $500 \mathrm{~mL}$ in 1 study) and balloon related trauma to the CS $[10,11,20]$.

Cardiac magnetic resonance (CMR) and MDCT scan are increasingly used to assess the anatomy of cardiac vessels, including cardiac veins. However, CMR cannot be used in patients with pacemakers or intracardiac defibrillators (encountered in $53.1 \%$ and $31.7 \%$, respectively in our study), and is less reliable than MDCT to depict cardiac vessel anatomy $[8,10,21]$.

Since the beginning of the 21 st century, many studies using MDCT have reported it as an easy, reliable and safe way to depict cardiac veins [7, 9-11, 22-25].

It has been demonstrated that 64 slices MDCT is a user-friendly and effective non-invasive method for visualizing cardiac veins before CRT [26, 27]. It can be used as a guide map for CRT [10].

In our study, we used 320 detectors MDCT to depict the CVS anatomy and to study the RA. Our study confirmed that the CS is an oval shaped structure, with a larger supero-inferior diameter, that requires a description in more than one dimension to know its real shape [7, 9].

We also demonstrated that there is a good correlation between echocardiography and MDCT for measuring the RA area and CS to RA lateral wall distance. There is also a good inter-observer agreement for RA and CS measurements. These findings may help the electrophysiologist to choose the appropriate catheter for cannulating the CS. Moreover, it may also demonstrate much dilated $\mathrm{RA}$, indicating a potentially difficult procedure using standard tools.

Our study showed that the CS and IIV are always present on MDCT $[7,9,10]$. In comparison to RV, MDCT has an excellent sensitivity to determine the presence of PV and LMV before CRT.

According to the IS and NIS sub-group analyses, no significant difference was demonstrated for the presence of PV or LMV regarding the IS or NIS status of the patient. Nevertheless, there was a tendency for the IS patients to present less LMV than NIS and our study may lack of power to appropriately confirm this finding. This would be in accordance with of the study of Van de Veire et al. [7], who concluded that patients with a history of infarction were less likely to have a LMV.

Finally, the recent EHRA/HRS consensus for CRT suggests, based in preliminary data, that knowledge of the CVS using MDCT could facilitate CRT though decreased procedure time and utilization of guiding catheters [12].

\section{Limitations of the study}

This is a retrospective study with a small sample size, therefore we consider it a very interesting study but it may have lacked power to confirm this finding and further investigations are necessary. This protocol did not include a follow-up of creatinine, renal failure or contrast nephropathy.

\section{Conclusions}

MDCT is as accurate as RV to depict CS and its tributaries (IIV, PV, LMV), and it could be useful as a non-invasive technique before CRT.

\section{Conflict of interest: None declared}

\section{References}

1. Epstein A.E, Di Marco JP, Ellembogen KA et al.; ACC/AHA/HRS 2008 Guidelines for Device-Based Therapy of Cardiac Rhythm Abnormalities: a report of the American College of Cardiology/ /American Heart Association Task Force on Practice Guidelines (Writing Committee to Revise the ACC/AHA/NASPE 2002 Guideline Update for Implantation of Cardiac Pacemakers and Antiarrhythmia Devices) developed in collaboration with the American Association for Thoracic Surgery and Society of Thoracic Surgeons. J Am Coll Cardiol, 2008; 51: 1-62.

2. Yancy CW, Jessup M, Bozkurt B et al.; American College of Cardiology Foundation; American Heart Association Task Force on Practice Guidelines. 2013 ACCF/AHA guideline for the management of heart failure: A report of the American College of Cardiology Foundation/American Heart Association Task Force on Practice Guidelines. J Am Coll Cardiol, 2013; 62: e147-239.

3. Abraham WT, Fisher WG, Smith AL et al. Cardiac resynchronization in chronic heart failure. N Engl J Med, 2002; 346: 1845-1853.

4. Yu CM, Gorcsan J 3rd, Bleeker GB et al. Usefulness of tissue doppler velocity and strain dyssynchrony for predicting left ventricular reverse remodeling response after cardiac resynchronization therapy. Am J Cardiol, 2007; 100: 1263-1270.

5. Merchant FM, Heist EK, Mc Carty D et al. Impact of segmental left ventricle lead position on cardiac resynchronization therapy outcomes. Hearth Rhythm, 2010; 7: 639-644.

6. Saremi F, Muresian H, Sanchez-Quintana D. Coronary Veins: Comprehensive CT-anatomic classification and review of variants and clinical implications. Radiographics, 2012; 32: 1-32.

7. Van de Veire NR, Schuijf JD, De Sutter J, Devos D, Bleeker GB, de Roos A. Non-invasive visualization of the cardiac venous system in coronary artery disease patients using 64-slice computed tomography. J Am Coll Cardiol, 2006; 48: 1832- 1838. 
8. Mühlenbruch G, Koos R, Wildberger JE, Gunther RW, Mahnken AHl. Imaging of the Cardiac Venous system: Comparison of MDCT and conventional angiography. Am J of Roentgenol, 2005; 185: 1252-1257.

9. Jongbloed MR, Lamb HJ, Bax JJ et al. Noninvasive visualization of the cardiac venous system using multislice computed tomography. J Am Coll Cardiol, 2005; 45: 749-753.

10. Doganay S, Karaman A, Gündogdu F, Duran C, Yalcin A, Kantarc M. Usefulness of multidetector computed tomography coronary venous angiography examination before cardiac resynchronization therapy. Jpn J Radiol, 2011; 29: 342-347.

11. Abbara S, Cury RC, Nieman K et al. Noninvasive evaluation of cardiac veins with MDCT angiography. Am J Roentgenol, 2005; 185: 1001-1006.

12. Daubert JC, Saxon L, Adamson PB et al. 2012 EHRA/HRS expert consensus statement on cardiac resynchronization therapy in heart failure: implant and follow-up recommendations and management. Heart Rhythm, 2012; 9: 1524-1576.

13. Singh JP, Houser S, Heist EK, Ruskin JN. The coronary venous anatomy. A segmental approach to aid cardiac resynchronization therapy. J Am Coll Cardiol, 2005; 46: 68-74.

14. Sa MI, de Roos A, Westenberg JJ, Kroft LJ. Imaging techniques in cardiac resynchronization therapy. Int J Cardiovasc Imag, 2006; 24: 89-105.

15. Puglisi A, Lunati M, Marullo AG et al. Limited thoracotomy as a second choice alternative to transvenous implant for cardiac resynchronization therapy delivery. Eur Heart J, 2004; 25:1063-1069.

16. Sinbane JS. Thebesian valve imaging with electron beam CT angiography: Implications for resynchronization theraphy. Pacing Clin Electrophysiol, 2004; 27: 1566-1567.

17. Habib A, Nirusha Lachman N, Christensen KN, Asirvatham S. The anatomy of the coronary sinus venous system for the cardiac electrophysiologist. Europace, 2009; 11: 15-21.
18. Meisel E, Pfeiffer D, Engelmann L et al. Investigation of coronary venous anatomy by retrograde venography in patients with malignant ventricular tachycardia. Circulation, 2001; 104: 442-447.

19. De Martino G, Messano L, Santamaria M et al. A randomized evaluation of different approaches to coronary sinus venography during biventricular pacemaker implants. Europace, 2005; 7: 73-76.

20. Meisel E, Butter C, Philippon F et al. Transvenous biventricular defibrillation. Am J Cardiol, 2000; 86: 76-85.

21. Chiribiri A, Kelle S, Götze S et al. Visualization of the cardiac venous system using cardiac magnetic resonance. Am J Cardiol, 2008; 101: 407-412.

22. Schaffler GJ, Groell R, Peichel KH, Rienmüller R. Imaging the coronary venous drainage system using electron-beam CT. Surg Rad Anat, 2000; 22: 35-39.

23. Gerber TC, Sheedy PF, Bell MR et al. Evaluation of the coronary venous system using electron beam computed tomography. Int J Cardiovasc Imag, 2001; 17: 65-75.

24. Mao S, Shinbane JS, Girsky MJ et al. Coronary venous imaging with electron beam computed tomographic angiography: Threedimensional mapping and relationship with coronary arteries. Am Heart J, 2005; 150: 315-22.

25. Tada H, Naito S, Koyama K, Taniguchi K. Three-dimensional computed tomography of the coronary venous system. J Cardiovasc Electrophysiol, 2003; 14: 1385.

26. Mlynarski R, Sosnowski M, Wlodyka A, Kargul W, Tendera M. User-friendly method of cardiac venous system visualization in 64-slice computed tomography. Pacing Clin Electrophysiol, 2009; 32: 323-329.

27. Hua W, Ding LG, Zhang S, Chen KP, Wang J, Wang FZ. Usefulness of previsualization of the cardiac venous system by 64 -slice computed tomography in patients with heart failure underwent cardiac resynchronization therapy. Zhonghua Xin Xue Guan Bing Za Zhi, 2010; 38: 610-613. 